\title{
INFLUÊNCIA DO SISTEMA DE PRODUÇÃO NA PRODUTIVIDADE DE LEITE NO INTERIOR DO ESTADO DE SÃO PAULO, BRASIL
}

\author{
João Lucas Cânovas Delfino ${ }^{1}$ \\ Thiago Luís Magnani Grassi ${ }^{2}$ \\ Elisa Helena Giglio Ponsano ${ }^{3}$ \\ Guilherme de Paula Nogueira ${ }^{3}$
}

\begin{abstract}
RESUMO
Esta pesquisa teve como objetivo avaliar a influência do sistema de produção sobre a produtividade de leite em propriedades rurais da microrregião de Birigui, São Paulo, Brasil. O estudo se baseou em coleta de dados dos sistemas de produção de leite da região citada, por meio de entrevista estruturada. Realizou-se amostragem por conveniência, sendo escolhidos 15 produtores de cada estrato produtivo (de acordo com a produção diária de leite: pequeno até $100 \mathrm{~L}$; médio - 101 a $300 \mathrm{~L}$; grande - mais de $300 \mathrm{~L}$ ), totalizando uma amostra com 45 produtores de leite. Apenas propriedades com base da alimentação volumosa em sistema de pastejo ou, no máximo, em semicofinamento participaram da pesquisa. Para avaliação da influência de diferentes fatores sobre a produtividade de leite, realizou-se regressão multivariada e análise de variância multivariada com pós-teste de Tukey. A produtividade verificada (média \pm desvio padrão), que representa a variável resposta do estudo, foi de 6.274,9 \pm 4.981,4 L/ha/ano. Com a análise estatística, pôde-se identificar a base da alimentação volumosa e a produção de leite por dia de intervalo entre partos como variáveis preditoras que influenciam a produtividade, sendo que a primeira foi a que exerceu maior influência sobre a variável resposta. Foi observado que não houve diferença $(p>0,05)$ entre a produtividade média das propriedades que utilizavam pastejo rotativo e das que forneciam volumoso no cocho, porém, em ambas, a produtividade foi superior $(\mathrm{p}<0,05)$ às médias de produtividade das propriedades que usavam pastejo contínuo e das que usavam pastejo alternado, sendo que estas não diferiram entre si ( $p>0,05)$. Em relação à base da alimentação volumosa, os resultados indicam a importância do manejo adequado de pastagens e demonstram a viabilidade da utilização, para produção de leite, de pastejo rotativo em vez de semiconfinamento. Quanto à produção de leite por dia de intervalo entre partos, os resultados denotam a importância da atenção à produção e à reprodução de forma conjunta, e não de forma isolada, para que haja eficiência produtiva no sistema. O foco do trabalho de produtores, técnicos, pesquisadores e outros participantes da cadeia produtiva do leite deve ser a sustentabilidade e o bem-estar animal.
\end{abstract}

Palavras-chave: gado leiteiro; pastejo; agricultura familiar; análise multivariada; sustentabilidade.

\footnotetext{
${ }^{1}$ Médico Veterinário. Universidade Estadual Paulista "Júlio de Mesquita Filho" - UNESP, jlcdelfino@ gmail.com

${ }^{2}$ Professor Doutor, Universidade Paulista (UNIP), Universidade do Oeste Paulista (UNOESTE) e Centro Universitário das Faculdades Integradas de Ourinhos. (UNIFIO) thiagograssi@ hotmail.com

${ }^{3}$ Professor(a) Adjunto, Departamento de Apoio, Produção e Saúde Animal, Faculdade de Medicina Veterinária, Universidade Estadual Paulista “Júlio de Mesquita Filho", UNESP, Campus de Araçatuba. elisahgp65@gmail.com.
} 


\title{
INFLUENCE OF PRODUCTION SYSTEM ON MILK PRODUCTIVITY IN INTERIOR OF SÃO PAULO STATE, BRAZIL
}

\begin{abstract}
This research aimed to evaluate the influence of the production system on milk productivity in farms from Birigui microregion, São Paulo, Brazil. The study was based on data collection from milk production systems in the aforementioned region, through a structured interview. Convenience sampling was carried out, with 15 producers chosen from each productive stratum (according to daily milk production: small - up to $100 \mathrm{~L}$; medium - 101 to $300 \mathrm{~L}$; large - more than $300 \mathrm{~L}$ ), totaling a sample with 45 milk producers. Only farms with grazing or semi-feedlot system for roughage feeding base were included. To evaluate the influence of different factors on milk productivity, multivariate regression and multivariate analysis of variance with Tukey's post-test were performed. The verified productivity (mean \pm standard deviation), which represents the study response variable, was 6,274.9 $\pm 4,981.4 \mathrm{~L} / \mathrm{ha} /$ year. With the statistical analysis, it was possible to identify roughage feeding base and milk production per days of calving interval as predictive variables that influence productivity, being roughage feeding base the one that exerted the greatest influence on the response variable. There was no difference in productivity between farms that used short-duration grazing system and roughage feeding base in the trough $(\mathrm{p}>0.05)$, but both had higher productivity $(\mathrm{p}<0.05)$ compared to farms that used continuous grazing system and alternated grazing system, which did not differ from each other ( $p>0.05)$. Regarding roughage feeding base, the results indicate the importance of adequate pasture management and demonstrate the feasibility of using, for milk production, rotational grazing instead of semi-feedlot. About milk production per days of calving interval, the results denote the importance of attention to production and reproduction together, and not in isolation, so that there is productive efficiency in the system. The focus of work from producers, technicians, researchers and other participants of milk production chain must be sustainability and animal welfare.
\end{abstract}

Keywords: dairy cattle; grazing; familiar agriculture; multivariate analysis; sustainability.

\section{INFLUENCIA DEL SISTEMA DE PRODUCCIÓN SOBRE LA PRODUCTIVIDAD DE LECHE EN EL INTERIOR DEL ESTADO DE SÃO PAULO, BRASIL}

\section{RESUMEN}

Esta investigación tuvo como objetivo evaluar la influencia del sistema de producción en la productividad de la leche en propiedades rurales en la microrregión de Birigui, São Paulo, Brasil. El estudio se basó en la recolección de datos de los sistemas de producción de leche en la región mencionada, mediante entrevista estructurada. Se realizó un muestreo de conveniencia con 15 productores de cada estrato productivo (según la producción diaria de leche: pequeño - hasta $100 \mathrm{~L}$; mediano - 101 a 300 L; grande - más de 300 L), un total de una muestra con 45 productores de leche. Solo participaron, en la investigación, propiedades basadas en alimentación voluminosa en sistema de pastoreo o, a lo sumo, en semi-encierro. Para evaluar la influencia de diferentes factores en la productividad de la leche, se realizó una regresión multivariada y un análisis de varianza multivariado con la prueba posterior de Tukey. La productividad verificada (media \pm desvío estándar), que representa la variable de respuesta del estudio, fue de 6.274,9 \pm 4.981,4 L/ha/año. Con el análisis estadístico, fue posible identificar la base de la alimentación voluminosa y la producción de leche por día de 
intervalo entre partos como variables predictoras que influyen en la productividad, siendo la primera la que ejerció la mayor influencia en la variable de respuesta. Se observó que no hubo diferencia $(p>0,05)$ entre la productividad media de las propiedades que usaban pastoreo rotativo y aquellas que proporcionaban voluminosos en el comedero, sin embargo, en ambas, la productividad fue mayor $(\mathrm{p}<0,05)$ a las medias de productividad de las propiedades que usaban pastoreo continuo y las que usaban pastoreo alterno, ya que no diferían entre sí $(\mathrm{p}>0,05)$. Con respecto a la base de la alimentación voluminosa, los resultados indican la importancia de un manejo adecuado de los pastos y demuestran la viabilidad del uso, para la producción de leche, del pastoreo rotativo en lugar del semi-encierro. En cuanto a la producción de leche por día de intervalo entre partos, los resultados denotan la importancia de la atención a la producción y la reproducción en conjunto, y no de forma aislada, de modo que haya una eficiencia productiva en el sistema. El enfoque del trabajo de los productores, técnicos, investigadores y otros participantes en la cadena de producción de leche debe ser la sostenibilidad y el bienestar animal.

Palabras clave: ganado lechero; pastoreo; agricultura familiar; análisis multivariante; sostenibilidad.

\section{INTRODUÇÃO}

Existem diversos tipos de sistemas de produção de leite no Brasil. Entretanto, no país, predominam aqueles cuja base da alimentação é o pasto (1). Segundo Lenzi (2), a nutrição deve continuar se baseando em pastagens, por ser a forma mais prática e econômica de alimentação de bovinos. Além disso, em vida livre, os animais desta espécie vivem em pastos, sem território fixo e com comportamento de grupo bem desenvolvido (3). Por isso, sistemas de produção a pasto consistem em ambientes mais próximos ao natural para bovinos, o que é importante para o bem-estar animal.

Produtores de leite de grande porte, majoritariamente, se dedicam somente ao produto em questão, enquanto que a maioria dos de pequeno porte desempenham outras atividades agrícolas (4). A diversificação das atividades é importante uma vez que a variedade de produções colabora para a sustentação das propriedades (5). Muitas vezes, em propriedades de pequeno porte, o conhecimento técnico é escasso. Pequenos produtores têm dificuldades com questões gerenciais, sanitárias, de manejo e capacidade de investimento (6). Assistência técnica de qualidade é indispensável para que o setor produtivo de leite tenha sustentabilidade (7).

Pesquisas de campo permitem que o cientista esteja em contato com o que ocorre no dia-a-dia dos produtores rurais. Trabalhos deste tipo possibilitam a obtenção de uma melhor noção do contexto da produção rural e das reais necessidades existentes no campo. A entrevista é um método de coleta de dados que pode ser utilizado em tais estudos. Segundo Manzini (8), a entrevista pode ser do tipo não-estruturada, semiestruturada e estruturada. A não-estruturada não tem um roteiro estabelecido de antemão, sendo iniciada por uma pergunta geradora, enquanto que, na semiestruturada, existe um roteiro prévio com perguntas abertas, devendo ser flexível a sequência de apresentação das perguntas (8). Já a entrevista estruturada é caracterizada por um roteiro prévio com perguntas fechadas e análise quantitativa, por meio dos conhecimentos da estatística (8).

O objetivo deste estudo foi avaliar a influência do sistema de produção sobre a produtividade de leite de propriedades rurais do interior do Estado de São Paulo, Brasil, por meio de coleta de dados com a utilização de entrevista estruturada. 


\section{MATERIAL E MÉTODOS}

Esta pesquisa foi submetida a apreciação ética, via sistema da Plataforma Brasil, e foi aprovada pelo Comitê de Ética em Pesquisa (CEP) da Faculdade de Odontologia da Universidade Estadual Paulista "Júlio de Mesquita Filho", campus de Araçatuba, sob o número 53191916.4.0000.5420 (parecer CEP 1.447.015).

Por meio de entrevista estruturada, a pesquisa se baseou em coleta de dados de sistemas de produção de leite da microrregião de Birigui, São Paulo, Brasil. Esta localidade apresenta clima tropical chuvoso com inverno seco, sendo que a precipitação do mês mais seco é inferior a $60 \mathrm{~mm}$ e a temperatura média do mês mais frio é superior a $18^{\circ} \mathrm{C}$ (9). A microrregião de Birigui está inserida na mesorregião de Araçatuba, Estado de São Paulo, possui 257.531 habitantes e uma área de $4.510,9 \mathrm{~km}^{2}$, sendo composta por 18 municípios (10).

Realizou-se amostragem por conveniência, devido à falta de informações sobre a população a ser abordada e à facilidade de contato com alguns dos produtores participantes e técnicos das Casas da Agricultura. A fim de buscar uma variação que permita possível inferência a outras regiões, escolheu-se 15 produtores de cada estrato (pequeno, médio e grande porte), totalizando uma amostra de 45 propriedades que produzem leite. Os estratos foram caracterizados de acordo com a produção diária de leite (11): pequeno produtor - até 100 litros; médio produtor - 101 a 300 litros; grande produtor - mais de 300 litros. Participaram da pesquisa apenas propriedades com base da alimentação volumosa em sistema de pastejo ou, no máximo, em cocho de semiconfinamento.

A partir dos dados coletados por entrevista estruturada, foi feita avaliação dos fatores que influenciam a produtividade de leite. A produtividade foi utilizada como variável resposta da pesquisa, sendo expressa como a quantidade de leite, em litros (L), produzida por ano dividida pela área útil da pecuária leiteira em hectares (ha), ou seja, litros/ha/ano. As variáveis preditoras numéricas são apresentadas na Tabela 1.

Tabela 1. Variáveis preditoras numéricas da pesquisa com 45 produtores de leite da microrregião de Birigui em relação à variável resposta "produtividade" (L/ha/ano)

\begin{tabular}{lc}
\hline \multicolumn{1}{c}{ Variável } & Unidade de medida \\
\hline Escrituração zootécnica ${ }^{(1)}$ & (não se aplica) \\
Vacinas não obrigatórias aplicadas & (não se aplica) \\
Tempo na atividade & Anos \\
Intervalo entre partos (IEP) médio do rebanho & Meses \\
Idade ao primeiro parto (IPP) média do rebanho & Meses \\
Produção de leite (PL) diária por vaca em lactação (VL) média do rebanho & Litros \\
PL por dia de IEP média do rebanho & Litros \\
Porcentagem de VL & $\%$ \\
Porcentagem de vacas no rebanho & $\%$ \\
Porcentagem de VL no rebanho & $\%$ \\
\hline
\end{tabular}

\footnotetext{
(1) 0 a 10, dependendo do número de respostas "sim" ao se perguntar se o produtor anotava cada uma das seguintes informações: data de cobertura, data de parição, controle leiteiro individual, produção de leite total, ficha individual das vacas, secagem das vacas, pesagem de fêmeas de reposição, calendário sanitário, despesas, receita.
}

Calcula-se a produção de leite (PL) por dia de intervalo entre partos (IEP) dividindo-se a produção de leite na lactação, em quilogramas (kg), pelo IEP, em dias (12). Considerando 
que existe divergência no método de mensuração da quantidade de leite (em L ou kg) entre os autores (7,13-16), optou-se pela medida em litros, já que o presente trabalho foi realizado com base em entrevistas e não faria diferença nas respostas o método de mensuração da produção de leite. A PL por dia de IEP pode ser calculada, também, dividindo-se a PL diária pelo total de vacas (lactantes e secas).

As variáveis preditoras categóricas para a variável resposta produtividade (L/ha/ano) são apresentadas na Tabela 2, sendo que foi atribuído um escore para cada resposta, o qual foi utilizado na análise estatística.

Tabela 2. Variáveis preditoras categóricas da pesquisa com 45 produtores de leite da microrregião de Birigui$\mathrm{SP}$ em relação à variável resposta produtividade (L/ha/ano) e seus respectivos escores

\begin{tabular}{lcccc}
\hline \multicolumn{1}{c}{ Variável } & \multicolumn{4}{c}{ Categorias } \\
\cline { 2 - 5 } & 1 & 2 & 3 & 4 \\
\hline Leite é atividade principal? & não & sim & (não se aplica) & (não se aplica) \\
Possui outras atividades? & não & sim & (não se aplica) & (não se aplica) \\
Assistência técnica & não tem & pública & particular & (não se aplica) \\
Grau de escolaridade ${ }^{(1)}$ & EF incompleto & EF completo & EM completo & ES completo \\
Família trabalha na atividade? & não & sim & (não se aplica) & (não se aplica) \\
Faz parte de associação? & não & sim & (não se aplica) & (não se aplica) \\
Base da alimentação volumosa & pastejo contínuo & pastejo alternado & pastejo rotativo ${ }^{(2)}$ & cocho \\
Divide vacas em lotes? & não & sim & (não se aplica) & (não se aplica) \\
Fornecimento de concentrado & não fornece & sem critério & por produção & (não se aplica) \\
Cobertura ${ }^{(3)}$ & somente MN & MC & IA e MN & somente IA \\
\hline
\end{tabular}

${ }^{(1)} \mathrm{EF}=$ ensino fundamental; $\mathrm{EM}=$ ensino médio; $\mathrm{ES}=$ ensino superior. ${ }^{(2)}$ foram considerados como pastejo rotativo apenas os sistemas que adotavam período de ocupação máximo de um dia por piquete. ${ }^{(3)} \mathrm{MN}=$ monta natural; $\mathrm{MC}=$ monta controlada; $\mathrm{IA}=$ inseminação artificial.

Para a análise estatística (17), com a finalidade de avaliar a influência de diferentes fatores sobre a produtividade de leite, foi utilizada estatística multivariada. Dentro desta técnica, foi realizada regressão multivariada e análise de variância multivariada com pós-teste de Tukey. Foi determinado, ainda, o coeficiente de correlação entre as variáveis numéricas. Para normalização dos dados, a variável resposta (produtividade) foi transformada em logaritmo. Além disso, a estatística descritiva foi feita para os dados obtidos na entrevista.

\section{RESULTADOS E DISCUSSÃO}

Foi possível identificar que a base da alimentação volumosa e a PL por dia de IEP foram as variáveis que influenciaram a produtividade (Tabela 4). Na Tabela 3, são apresentados média, desvio padrão, mínimo e máximo dos índices produtivos das propriedades incluídas neste trabalho.

A produtividade média encontrada nesta pesquisa (Tabela 3) foi numericamente maior $(6.274,9 \pm 4.981,4 \mathrm{~L} / \mathrm{ha} / \mathrm{ano})$ do que aquela encontrada por Oliveira et al. (15) em propriedades leiteiras do Extremo Sul da Bahia $(895,28 \pm 383,97 \mathrm{~L} / \mathrm{ha} / \mathrm{ano})$, nas quais a pastagem é o volumoso exclusivo das águas. No estudo de Lopes et al. (14), com propriedades leiteiras de Minas Gerais, foi observada produção de leite de 6.818,31 \pm $1.756,51 \mathrm{~kg} / \mathrm{ha} /$ ano para semiconfinamento, sendo este resultado semelhante ao encontrado na presente pesquisa. 
Ferreira e Miranda (12) consideram que a produtividade da terra deve ser superior a 20 $\mathrm{L} / \mathrm{ha}$ /dia (rebanhos mestiços em pasto de braquiária), que equivale a cerca de $7.300 \mathrm{~L} / \mathrm{ha} / \mathrm{ano}$, limite que está acima da média observada na presente pesquisa $(17,2 \mathrm{~L} / \mathrm{ha} / \mathrm{dia})$. Contudo, é importante salientar que existem diferenças regionais e, com isso, parâmetros podem diferir de acordo com a localidade em questão. Em estudo de caso com propriedades de Minas Gerais, as quais recebiam assistência técnica e econômica pelo Programa de Desenvolvimento da Pecuária de Leite da Região de Viçosa, foi relatado, em uma propriedade, que a elevação da produtividade de 324,87 L/ha/ano para 3.467,89 L/ha/ano, em um período de 23 anos, resultou em aumento da rentabilidade do sistema de produção, que se tornou competitivo (7).

Tabela 3. Média, desvio padrão, mínimo e máximo das variáveis numéricas da pesquisa com 45 produtores de leite da microrregião de Birigui-SP

\begin{tabular}{|c|c|c|c|c|}
\hline Variável & Média & Desvio padrão & Mínimo & Máximo \\
\hline Produtividade (L/ha/ano) & $6.274,9$ & $4.981,4$ & 506,9 & $23.227,3$ \\
\hline $\operatorname{IEP}^{(1)}($ meses $)$ & 13,1 & 1,3 & 11,5 & 18,0 \\
\hline $\operatorname{IPP}^{(2)}$ (meses) & 33,2 & 3,7 & 24,0 & 42,0 \\
\hline $\mathrm{PL}^{(3)}$ diária $(\mathrm{L})$ & 287,2 & 281,3 & 40,0 & $1.350,0$ \\
\hline PL diária por $\mathrm{VL}^{(4)}(\mathrm{L})$ & 10,9 & 4,8 & 3,2 & 21,8 \\
\hline PL por dia de IEP $(\mathrm{L})$ & 7,8 & 3,9 & 2,2 & 19,3 \\
\hline VL $(\%)$ & 71,0 & 14,2 & 33,3 & 95,1 \\
\hline Vacas no rebanho $(\%)$ & 58,3 & 15,9 & 32,3 & 100,0 \\
\hline VL no rebanho (\%) & 41,4 & 14,0 & 14,5 & 73,7 \\
\hline Tempo na atividade (anos) & 18,9 & 11,8 & 2,0 & 48,0 \\
\hline Escrituração zootécnica $^{(5)}$ & 5,6 & 2,5 & 0,0 & 10,0 \\
\hline Vacinas não obrigatórias aplicadas & 1,1 & 0,8 & 0,0 & 3,0 \\
\hline
\end{tabular}

Os $71,0 \pm 14,2 \%$ de vacas em lactação (VL) observados neste estudo estão próximos do índice de $75 \%$ considerado, por Ferreira e Miranda (12), como bom para sistemas de produção de leite a pasto ou em semiconfinamento. Considera-se que 12 meses é um IEP ideal, principalmente para rebanhos mestiços, podendo-se adotar 14 meses para a raça Holandesa (18). A presença desta raça foi pouco observada nas propriedades avaliadas nesta pesquisa, onde foi observado predomínio de animais da raça Girolando e IEP $(13,1 \pm 1,3$ meses) próximo do ideal. De maneira semelhante, a IPP de 33,2 \pm 3,7 meses (Tabela 3) assemelhou-se aos 30 a 32 meses que Ferreira e Miranda (12) consideram ideal para fêmeas mestiças. Conforme apresentado na Tabela 3 , a porcentagem média de vacas no rebanho $(58,3$ $\pm 15,9 \%$ ) esteve acima da composição tida como ideal de 50\% (18), enquanto que a porcentagem média de VL no rebanho $(41,4 \pm 14,0 \%)$ esteve próxima dos $42 \%$ considerados ideais (18). De modo geral, observa-se que os rebanhos estudados, em média, apresentaram características próximas do que é considerado ideal na literatura. Nesse contexto, é importante ressaltar que cada propriedade apresenta suas particularidades, com certas semelhanças regionais. Assim, deve-se considerar que os diferentes sistemas de produção podem alcançar o equilíbrio de formas diferentes.

A PL por dia de IEP foi de 7,8 \pm 3,9 L (Tabela 3). Segundo Ferreira e Miranda (12), este parâmetro representa o melhor índice para se medir a eficiência da atividade leiteira. Por

Delfino JLC, Grassi TLM, Ponsano EHG, Nogueira GP. Influência do sistema de produção na produtividade de leite no interior do estado de São Paulo, Brasil. Vet. e Zootec. 2020.; 27: 001-011. 
isso, é mais esclarecedora que a PL diária por VL. Certos fatores, como a base genética, podem influenciar a PL por dia de IEP. Foram observados maiores valores para este índice $(\mathrm{p}<0,05)$ em fêmeas de base genética materna Gir $(5,06 \pm 1,38 \mathrm{~kg})$ em comparação com fêmeas de base genética materna Guzerá $(4,37 \pm 1,68 \mathrm{~kg})$, em estudo com novilhas F1 Holandês x Zebu (16), valores inferiores à média observada neste trabalho.

As vacinas não obrigatórias citadas durante as entrevistas foram contra clostridioses, paratifo, leptospirose, ceratoconjuntivite, rinotraqueíte infecciosa bovina e diarreia viral bovina. A escrituração zootécnica mais frequente foi a data de parição, realizada por $88,9 \%$ (40/45) dos produtores entrevistados, enquanto que a menos frequente foi a pesagem de fêmeas de reposição, feita por apenas 8,9\% (4/45) dos produtores.

O leite era a atividade principal para 73,3\% (33/45) dos produtores entrevistados. A assistência técnica era exclusivamente pública para 53,4\% (24/45) dos produtores, particular para 22,2\% (10/45) e 24,4\% (11/45) não recebiam assistência técnica. Outras atividades, como agricultura, bovinocultura de corte, pesca e emprego formal, eram desempenhadas por $73,3 \%$ (33/45) dos entrevistados.

O grau de escolaridade variou entre ensino fundamental incompleto $(37,7 \%$ - 17/45), ensino fundamental completo $(8,9 \%$ - 4/45), ensino médio completo $(35,6 \%$ - 16/45) e ensino superior completo $(17,8 \%$ - 8/45). A família trabalhava diretamente na atividade em $77,8 \%$ (35/45) das propriedades visitadas e 64,4\% (29/45) dos produtores faziam parte de algum tipo de associação.

Do total de produtores, 28,9\% (13/45) separava as vacas em lotes. Apenas 6,7\% (3/45) dos entrevistados não fornecia concentrado ao rebanho, enquanto 22,2\% (10/45) fornecia sem critério e $71,1 \%$ (32/45) fornecia de acordo com a produção das vacas. A base da alimentação volumosa mais observada foi pastejo rotativo $(51,2 \%$ - 23/45) seguido de pastejo contínuo $(22,2 \%$ - 10/45), pastejo alternado $(13,3 \%$ - 6/45) e fornecimento no cocho $(13,3 \%-6 / 45)$. Para a reprodução, 59,9\% (27/45) dos produtores utilizava somente monta natural (MN), $6,7 \%$ (3/45) monta controlada, 15,6\% (7/45) MN e inseminação artificial (IA) e 17,8\% (8/45) somente IA.

Por meio da estatística multivariada, foi possível avaliar, de forma conjunta, a influência das variáveis preditoras sobre a variável resposta. A regressão multivariada demonstrou que a produtividade (variável resposta) foi influenciada por duas variáveis preditoras: base da alimentação volumosa e PL por dia de IEP (Tabela 4).

Tabela 4. Análise de modelos de regressão multivariada da produtividade com a base da alimentação volumosa e produção de leite (PL) por dia de intervalo entre partos (IEP) da pesquisa com 45 produtores de leite da microrregião de Birigui-SP

\begin{tabular}{clccc}
\hline Variável resposta & \multicolumn{1}{c}{ Variável preditora } & Valor estimado & Desvio padrão & $\mathrm{P}$ \\
\hline \multirow{2}{*}{ Produtividade } & Intercepto & 6,89897 & 0,28881 & $<0,0001$ \\
(L/ha/ano) & Base da alimentação volumosa & 0,23666 & 0,10835 & 0,0346 \\
& PL por dia de IEP & 0,11763 & 0,02767 & 0,0001 \\
\hline
\end{tabular}

A análise de variância multivariada demonstrou que as mesmas variáveis preditoras, base da alimentação volumosa $(p=0,0170)$ e PL por dia de IEP $(p=0,0002)$, exercem influência sobre a produtividade. A partir dos resultados, nota-se a importância da nutrição do rebanho para o desempenho produtivo. A base da alimentação volumosa foi a variável que exerceu maior influência na produtividade. Observou-se que as produtividades médias das propriedades que utilizavam pastejo rotativo e fornecimento no cocho não diferiram entre si $(\mathrm{p}>0,05)$. Entretanto, foram superiores $(\mathrm{p}<0,05)$ às produtividades médias das propriedades que utilizavam pastejo contínuo e pastejo alternado, que também não diferiram entre si $(p>0,05)$. Esses resultados evidenciam a importância de se manejar adequadamente as

Delfino JLC, Grassi TLM, Ponsano EHG, Nogueira GP. Influência do sistema de produção na produtividade de leite no interior do estado de São Paulo, Brasil. Vet. e Zootec. 2020.; 27: 001-011. 
pastagens e demonstram a viabilidade do uso de pastejo rotativo em vez de alimentação volumosa baseada em fornecimento no cocho.

Foi observado aumento na produtividade de $6.443 \mathrm{~L} / \mathrm{ha}$ ao ano em 2009 para $9.467 \mathrm{~L} / \mathrm{ha}$ ao ano em 2012, além do aumento na lucratividade, ao se utilizar o manejo de pastagem em propriedade de gado leiteiro do Estado de São Paulo (19). Observou-se, em estudo de rentabilidade de sistemas de produção de leite na região de Lavras (MG), que a alimentação é o item que exerce maior influência no custo operacional efetivo (20). Em trabalho com sistemas intensivos de produção de leite em Minas Gerais, sistemas de semiconfinamento apresentaram viabilidade econômica com condições de produzir em curto, médio e longo prazo, capitalizando os produtores, enquanto que sistemas de confinamento total apresentaram margem bruta negativa, causando descapitalização e endividamento dos produtores (14). A análise dos resultados acima induz a crer que, quanto menos se utiliza pastagens, menos rentável o sistema se torna.

Se manejada intensivamente, a pastagem representa o volumoso de melhor qualidade, podendo ser produzido a um custo baixo (21). Em comparação com a alimentação baseada em fornecimento de volumoso no cocho, o uso intensivo de pastagens pode reduzir os gastos com mão-de-obra, pois a colheita do alimento é feita pelo próprio animal. A propriedade estudada por Costa et al. (13) apresentava rentabilidade utilizando alimentação, nos meses de verão, a base de pastagens naturais e suplementação com concentrado e, nos meses de inverno, baseada em silagem de milho ou cana-de-açúcar com concentrado.

Além disso, é essencial considerar a questão do bem-estar animal, uma vez que, em sistemas de produção a pasto, os bovinos encontram-se em ambiente mais próximo do natural para sua espécie. Nesse contexto, é preciso que os animais sejam adaptados às condições ambientais em questão. Costa et al. (13) observaram que vacas com grau de sangue 1/2 e 1/4 Holandês-Zebu, no Norte de Minas Gerais, em uma localidade de clima tropical com inverno seco e verão chuvoso, podem constituir uma alternativa mais lucrativa para sistemas de produção que utilizam mais pastagens e menos tecnificação. Segundo Ferreira et al. (22), comparadas a raças que evoluíram em clima temperado, aquelas de origem indiana são mais resistentes às condições tropicais. Estes autores relatam, ainda, que é importante o conforto térmico de bovinos leiteiros a pasto em ambiente tropical, para que a produção de leite e a reprodução mantenham-se adequadas.

A PL por dia de IEP associa fatores relacionados a produção e reprodução (12). Detectada pela regressão multivariada (Tabela 4), a influência da PL por dia de IEP sobre a produtividade denota a importância da atenção à produção e à reprodução de forma conjunta, para que haja eficiência produtiva do sistema. Por exemplo, uma vaca altamente produtiva pode ter problemas reprodutivos, além de possivelmente ter a rusticidade prejudicada. Nesse sentido, o animal deve ser considerado como um ser senciente que possui diversos atributos, não apenas os que estão diretamente relacionados à produção. Costa et al. (13) verificaram, em sua pesquisa com informações de um rebanho do Norte de Minas Gerais, que a mais alta produtividade de animais com maior proporção genética da raça holandesa não se traduz em maior rentabilidade, devido ao maior custo de produção.

Observou-se rentabilidade e competitividade em estudo de caso de propriedade com produção por total de vacas (PL por dia de IEP) de 7,69 L, onde os animais tinham grau de sangue que variava de 3/4 a 7/8 Holandês-Zebu (7), valor semelhante aos 7,8 $\pm 3,9 \mathrm{~L}$ observados na presente pesquisa. Em seu estudo, Costa et al. (13) verificaram que a rentabilidade apresentou tendência inversa da produção de leite, sendo que o acréscimo de animais com composição racial 1/4 e 1/2 Holandês-Zebu fez com que a rentabilidade do sistema aumentasse de $10 \%$ para $18 \%$ em cinco anos. Nesse sentido, é interessante pontuar que produção máxima não é sinônimo de maior lucro, é preciso encontrar o equilíbrio. A produtividade por total de vacas e a produtividade da terra (L/ha/ano), dentre outros 
indicadores, apresentaram correlação positiva com a taxa de remuneração do capital investido, em pesquisa com produção de leite no Extremo Sul da Bahia (15). Esses resultados indicam a importância tanto da produtividade quanto da PL por dia de IEP na rentabilidade da atividade leiteira.

O sistema de produção de leite deve ser visto como um todo, de forma que seus setores devem estar em equilíbrio, independentemente se o produtor realiza outras atividades ou não. Cada propriedade apresenta uma realidade peculiar, com certas similaridades regionais. Nesse contexto, assistência técnica e extensão rural de qualidade são de extrema importância para a rentabilidade do sistema. O trabalho de produtores, técnicos, pesquisadores e outros participantes da cadeia produtiva deve ter como foco a sustentabilidade e o bem-estar animal.

\section{CONCLUSÕES}

Sistemas de produção de leite baseados em pastejo rotativo apresentaram maior produtividade (L/ha/ano), chegando a não diferirem de sistemas com base de alimentação volumosa no cocho (semiconfinamento), em comparação com sistemas baseados em pastejo contínuo ou pastejo alternado. A produção de leite por dia de intervalo entre partos influenciou positivamente a produtividade.

\section{AGRADECIMENTOS}

À Coordenação de Aperfeiçoamento de Pessoal de Nível Superior (CAPES), pela bolsa concedida no início do mestrado. À Fundação de Apoio a Pesquisa, Ensino e Extensão, pelo apoio financeiro à pesquisa (projeto FUNEP número 3170). A todos que colaboraram para que o projeto se tornasse realidade.

\section{REFERÊNCIAS}

1. Silva JJ, Carvalho DMG, Gomes RAB, Rodrigues ABC. Produção de leite de animais criados em pastos no Brasil. Vet Zootec. 2010;17(1):26-36.

2. Lenzi A. Fundamentos do Pastoreio Racional Voisin. Rev Bras Agroecol. 2012;7(1):82-94.

3. Molento CFM, Bond GB. Aspectos éticos e técnicos da produção de bovinos. In: Anais do Congresso Brasileiro de Bioética e Bem-Estar Animal; 2008, Recife. Recife: Conselho Federal de Medicina Veterinária; 2008. p.43-8.

4. Ribeiro Junior JC, Tamanini R, Silva LCC, Beloti V. Quality of milk produced by small and large dairy producers. Semina Cienc Agrar. 2015;36(2):883-8.

5. Hunt D, Shiki S, Ribeiro R, Biasi D, Faria AP. Comparação de indicadores de desempenho de produtores de leite localizados dentro e fora de assentamentos de reforma agrária no Triângulo Mineiro. Rev Econ Sociol Rural. 2009;47(1):211-48.

6. Menezes CCP, Martines L, Pagani Netto C. Projeto CATI Leite. In: Pagani Netto C, coordenador. CATI Leite. Campinas: CATI; 2012. p.1-12

7. Silva MF, Pereira JC, Gomes ST, Nascif C, Gomes AP. Avaliação dos indicadores zootécnicos e econômicos em sistemas de produção de leite. Rev Polit Agric. 2015;24(1):62-73.

Delfino JLC, Grassi TLM, Ponsano EHG, Nogueira GP. Influência do sistema de produção na produtividade de leite no interior do estado de São Paulo, Brasil. Vet. e Zootec. 2020.; 27: 001-011. 
8. Manzini EJ. Uso da entrevista em dissertações e teses produzidas em um programa de pósgraduação em educação. Percurso. 2012;4(2):149-71.

9. Miranda MJ, Pinto HS, Zullo Júnior J, Fagundes RM, Fonsechi DB, Calve L, et al. Clima dos municípios paulistas: a classificação climática de Koeppen para o estado de São Paulo [Internet]. Campinas: Unicamp; 2015 [cited 2016 Maio 23]. Available from: http://www.cpa.unicamp.br/outras-informacoes/climados-municipios-paulistas.html

10. Instituto Brasileiro de Geografia e Estatística. Censo demográfico 2010 [Internet]. Rio de Janeiro: IBGE; 2010 [cited 2016 Jul 16]. Available from: http://www.sidra.ibge.gov.br/cd/cd2010sp.asp?o=3\&i=P

11. Furlaneto FPB, Esperancini MST, Salla DA. Caracterização do sistema produtivo de leite na região do Médio Paranapanema, Estado de São Paulo. In: Anais do Congresso da Sociedade Brasileira de Economia, Administração e Sociologia Rural; 2008, Rio Branco. Rio Branco: Sociedade Brasileira de Economia, Administração e Sociologia Rural; 2008. p.1-11

12. Ferreira AM, Miranda JEC. Medidas de eficiencia da atividade leiteira: índices zootecnicos para rebanhos leiteiros. Juiz de Fora: EMBRAPA; 2007. (Comunicado técnico, n. 54).

13. Costa MD, Ruas JMN, Martins Neto T, Rodriguez MAP, Venturin RP, Mendes LJ, et al. Análise da relação benefício-custo em sistema de produção de leite com animais mestiços no Norte de Minas Gerais. Bol Ind Anim. 2016;73(3):244-51.

14. Lopes MA, Santos G, Carvalho FM. Comparativo de indicadores econômicos da atividade leiteira de sistemas intensivos de produção de leite no Estado de Minas Gerais. Rev Ceres. 2012;59(4):458-65.

15. Oliveira AS, Cunha DNFV, Campos JMS, Vale SMLR, Assis AJ. Identificação e quantificação de indicadores-referência de sistemas de produção de leite. Rev Bras Zootec. 2007;36(2):507-16.

16. Ruas JRM, Carvalho BC, Silva Filho JM, Silva MA, Palhares MS, Brandão FZ. Efeito da base genética materna e da estação de parição sobre variáveis produtivas de fêmeas primíparas Holandês x Zebu. Arq Bras Med Vet Zootec. 2007;59(1):218-24.

17. SAS Institute Inc. The SAS System, release 9.3. Cary: SAS; 2016.

18. Campos AT, Ferreira AM. Composição do rebanho e sua importância no manejo. Juiz de Fora: EMBRAPA; 2006.

19. Gonçalves ACS, Roma Júnior LC, Fonseca MI, Nadruz BV, Bürger KP, Rossi GAM. Assistência técnica e extensão rural: sua importância para a melhoria da produção leiteira. Relato de caso. Rev Bras Hig Sanidade Anim. 2014;8(3):47-61. 
20. Lopes MA, Lima ALR, Carvalho FM, Reis RP, Santos IC, Saraiva FH. Controle gerencial e estudo da rentabilidade de sistemas de produção de leite na região de Lavras (MG). Cienc Agrotec. 2004;28(4):883-92.

21. Dalpian T, Stivari A, Avelar PCB, Pagani Netto C. Implantação, manejo e adubação das pastagens. In: Pagani Netto C, coordenador. CATI Leite. Campinas: CATI; 2012. p.25-88

22. Ferreira IC, Martins CF, Fonseca Neto AM, Cumpa HCB. Conforto térmico em bovinos leiteiros a pasto. Planaltina: EMBRAPA; 2017. (Documentos, n. 342).

Recebido em: 16/08/2020

Aceito em: 19/11/2020 